\title{
Quantum-chemical free energy calculation of vinpocetine molecule release from sodium alginate
}

\author{
Yulia POLKOVNIKOVA ${ }^{1 *}$, Kseniya KORYANOVA ${ }^{2}$, Halahakoon Amila JEEWANTHA ${ }^{1}$
}

1 Federal State Budget Educational Institution of Higher Education "Voronezh State University," 394036, Voronezh, ul. Student, 3, Russia.

2 Pyatigorsk Medical and Pharmaceutical Institute - branch of FGBOU in VolgGMU Ministry of Healthcare of Russia, 357500, Pyatigorsk, Kalinin Ave., 11, Russia.

* Corresponding Author. E-mail: juli-polk@mail.ru (Y.P.); Tel. +79081397582; ORCID No: 0000-0003-0123-9526.

Received: 17 October 2017 / Revised: 07 February 2018 / Accepted: 12 February 2018

\begin{abstract}
Microencapsulation is a method of obtaining traditional materials in a unique form that is able to protect substances from the effects of environmental factors that cause their decomposition. This method is used in medicine and pharmacy, where drugs and enzymes are encapsulated in biodegradable microcapsules. The aim of the study was a possibility of the use of sodium alginate for microencapsulation of vinpocetine on the basis of comparative analysis of thermodynamic characteristics concerned with the efficiency of vinpocetine release from sodium alginate into water and ethanol. The following methods of investigations were applied: The molecular modeling of the process of vinpocetine release from the microcapsules into various media was carried out for: water with $\mathrm{pH}$ value $2.0,7.0$, and ethyl alcohol 95\%. The molecular dynamics method in the Bioevrik program was implemented to the modeling "polymer-vinpocetine" systems in the aqueous and ethanol environments. Results of the study: It was found that vinpocetine release is mostly energy-efficient into aqueous medium with $\mathrm{pH}=2$, as compared with aqueous medium at $\mathrm{pH}=7$ and $95 \%$ ethyl alcohol.
\end{abstract}

KEYWORDS: Microcapsules; vinpocetine; sodium alginate; molecular dynamics; quantum chemistry.

\section{INTRODUCTION}

The scientific and practical interest in the problem of microencapsulation still remains high, as evidenced by the extensive literature search on this topic. A set of polymers as a carrier of an active substance in microencapsulation is determined by the required rate of their release, that to a large extent depends on the physical properties of the polymer [[1]]. The water-soluble and biodegradable polymer, sodium alginate is often used as a coating material [[2],3]. The drugs encapsulated in a shell of sodium alginate are much more resistant to the environmental factors and capable of forming stable nanodispersed suspensions, which are visually indistinguishable from true solutions $(\sim$ particle size $<1.0 \mathrm{~nm})$. They can serve as a source for the new dosage forms [[4],5].

The microcapsules with a sodium alginate shell are predominantly obtained by an extrusion method. However, quite often the complication of the instruments design and low efficiency make the selection of microencapsulation technique rather long and costly process.

Recently an ether of $(3,16)$-eburnamenin-14-carboxylic acid has been widely recognized in the medical practice. It is applied in the cases of stroke of different nature. It has vasodilating, antihypoxic and antiaggregate effect.

Vinpocetine is produced as a peroral dosage pharmeceutical form - pills. The main drawbacks of these forms are their weakly expressed action.

A promising drug for the designed a microencapsulated form is Vinpocetine [6,7].

Vinpocetine is poorly water soluble. One can use purified water, solution of hydrochloric acid as a solvent medium for the conducting of preliminary studies for the rationale of the composition of microcapsules and for determining the conditions of their preparation. In addition, ethyl alcohol can be used, which makes it possible to determine more distinctly the effect of the excipients and technological factors [8].

How to cite this article: Polkovnikova Y, Koryanova K, Jeewantha HA. Quantum-chemical free energy calculation of vinpocetine molecule release from sodium alginate. Marmara Pharm J. 2018; 22 (3): 443-449. 
Modeling the molecular dynamics of solutions in the study of the drug release process from the microcapsules allows selecting the optimal film former, the $\mathrm{pH}$ index, the release medium and other factors essential for the successful microencapsulation process. These are minimal requirements for the real experiment. Theoretical modeling makes it possible to reduce the number of conducted experiments and to optimize the way of solving practical problems. It should be also noted the possibility of prredicting the release of medicinal substances into various media $[9,10]$.

The modern chemical theory allows a high degree of reliability to provide a numerical prediction of the possibility of the existence for a given molecular system and its individual parameters (spatial structure, electron density distribution in a molecule, and so forth). Moreover, many of the properties of molecules that are difficult (or even impossible) to determine experimentally, become available for the description [11]. Using the methods of quantum chemistry it is possible to conclude the prospects and the necessity to use sodium alginate as a microcapsules shell for vinpocetine.

The studies carried out at the previous stage of molecular dynamics modeling of vinpocetine release from sodium alginate showed the possibility of vinpocetine release into the water with $\mathrm{pH}-2.0$ and ethanol. The obtained results demonstrated a lower degree of vinpocetine release from sodium alginate into ethanol medium as compared to the $0.01 \mathrm{M} \mathrm{HCl}$ solution.

At the same time, the molecular dynamics method, based on the use of molecular mechanical fields, is a more approximate method of molecular modeling than the method of quantum chemistry $[12,13]$. Basing on this idea the calculation of thermodynamic characteristics of the release from the polymer by the quantum chemical method makes it possible to estimate more reliably the affinity of vinpocetine to sodium alginate. The data were obtained from the studies allow making a comparative conclusion about the efficiency of vinpocetine release and the applications possibility of sodium alginate for the microencapsulation purpose.

The purpose of the study:

The principal goal of this work is the prediction for the application possibility of sodium alginate for vinpocetine microencapsulation, based on the comparative analysis of thermodynamic characteristics of the vinpocetine release efficiency from the sodium alginate into aqueous and ethanol medium.

Objectives of the study:

1. Prediction of the spatial structures of the complexes "vinpocetine- sodium alginate " in water at lower $\mathrm{pH}$ index - $\mathrm{pH}$ 2.0, middle - $\mathrm{pH} 7$ and in ethanol basing on the results of molecular dynamics modeling.

2. The assignment of the systems fragments of "vinpocetine-alginate," "vinpocetine-water," "vinpocetineethanol" available for the quantum-chemical modeling.

3. Conducting an oscillatory analysis of the obtained molecular models for determining the entropy, enthalpy and Gibbs energy of vinpocetine release into solvents medium - water and ethanol.

\section{RESULTS}

In order to make quantum-chemical calculation of thermodynamic characteristics, the fragments of the initial (vinpocetine-polymer, solvent) and final (polymer, vinpocetine-solvent) states were discriminated from the systems used for the molecular dynamics modeling. The resulted molecular models were used for the quantum-chemical calculation of thermodynamic characteristics of the process of vinpocetine release from sodium alginate [14,15].

Figure 1 shows the structure of molecular models for the system states when vinpocetine is released from the polymer into the water into acidic medium ( $\mathrm{pH} 2)$. As initial condition, vinpocetine was examined in contact with a fragment of alginic acid and water molecules, as well as a solvent (water). The final state is represented by a fragment of sodium alginate in contact with water molecules and a molecule of vinpocetine with a solvated shell. Vinpocetine is introduced into the simulated system as a cation in conformity with the $\mathrm{pH}$ value of $\mathrm{pH} 2$.

Figure 2 shows the structure of molecular models for the system states when vinpocetine is released from the polymer into water that is a neutral medium ( $\mathrm{pH} 7)$. As the initial condition, vinpocetine was used in contact with a sodium alginate fragment and water molecules, as well as a solvent (water). The final state is represented by a sodium alginate fragment in contact with water molecules and a molecule of vinpocetine with a solvated shell. Vinpocetine is introduced into the simulated system in the form of the base in conformity with the $\mathrm{pH}$ value of $\mathrm{pH} 7$. 


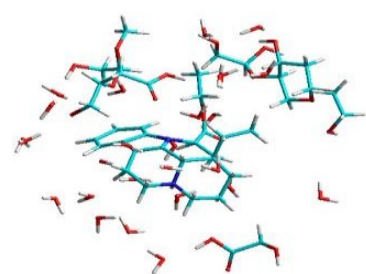

Vinpocetine in alginic acid

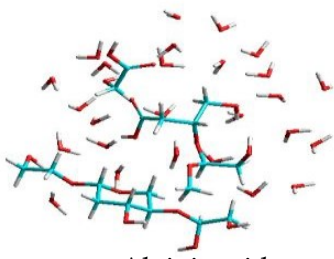

Alginic acid
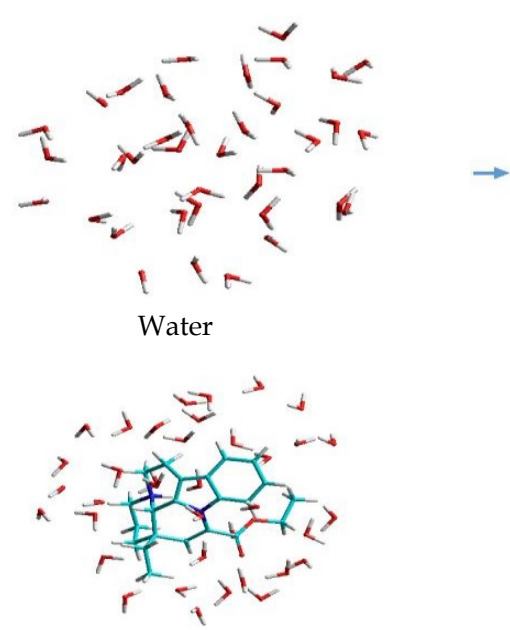

Vinpocetine in the water

Figure 1. The release of vinpocetine from alginic acid into water at $\mathrm{pH} 2$.

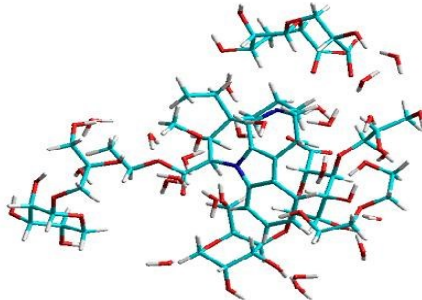

Vinpocetine in sodium alginic

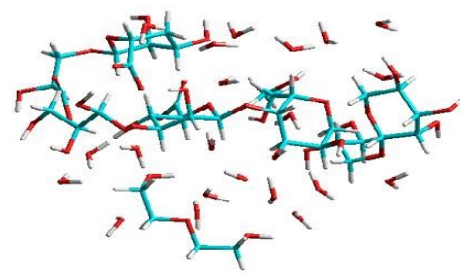

Sodium alginic

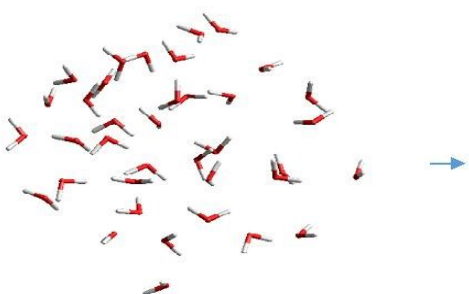

Water

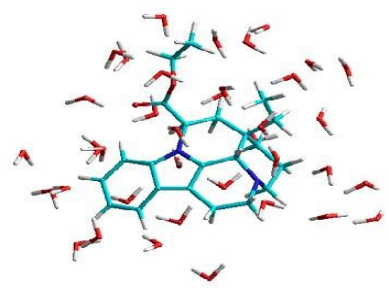

Vinpocetine in the water

Figure 2. The release of vinpocetine from sodium alginate into water at $\mathrm{pH} 7$.

Figure 3 shows the structure of molecular models of system states when vinpocetine is released from the polymer into ethanol medium. As an initial condition, vinpocetine is used in contact with a fragment of sodium alginate and ethanol molecules, as well as a fragment of a solvent (ethanol). The final state is represented by a fragment of alginate, in contact with ethanol molecules and a molecule of vinpocetine with a solvate shell. Vinpocetine is included in the simulated system in the form of a base.

Quantum-chemical calculations of enthalpy, entropy, and entropy of Gibbs for vinpocetine released from the polymer into a solvent were performed to determine the affinity of vinpocetine to sodium alginate into the different medium (ethanol and water) at the different values of hydrogen index ( $\mathrm{pH} 2$ and $\mathrm{pH} 7$ ) $[16,17]$. Enthalpy, entropy, and Gibbs energy were calculated as the difference between the data of thermodynamic functions of the final and initial states of the system. The results of calculating thermodynamic characteristics of vinpocetine release are presented in Table 1-3. 


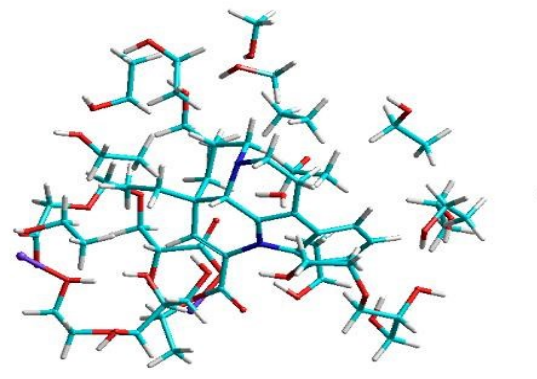

Vinpocetine in sodium alginic

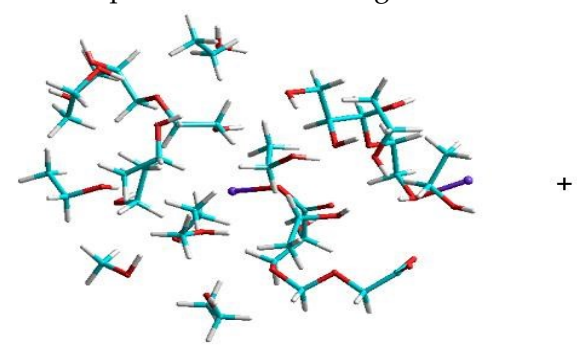

Sodium alginic

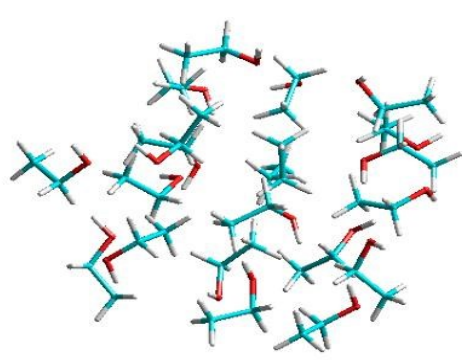

Ethanol

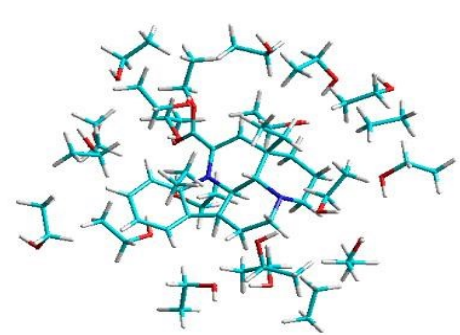

Vinpocetine in ethanol

Figure 3. The release of vinpocetine from sodium alginate into the ethanol.

Table 1. The results of quantum-chemical calculation for the release enthalpy of vinpocetine from sodium alginate into solvent.

\begin{tabular}{|c|c|c|c|c|c|}
\hline \multirow[b]{2}{*}{ System } & \multicolumn{3}{|c|}{ Enthalpy of the system, $\mathbf{k J} / \mathbf{m o l}$} & \multirow[b]{2}{*}{$\begin{array}{l}\text { Vinpocetine in the } \\
\text { solvent }\end{array}$} & \multirow{2}{*}{$\begin{array}{l}\text { Released enthalpy } \\
\text { kJ/mol }\end{array}$} \\
\hline & $\begin{array}{l}\text { Vinpocetine in } \\
\text { polymer }\end{array}$ & Solvent & $\begin{array}{l}\text { Polymer without } \\
\text { vinpocetine }\end{array}$ & & \\
\hline $\mathrm{pH}=2(1)$ & -12916834.42 & -7284492.27 & -10044950.29 & -10156853.84 & -477.44 \\
\hline $\mathrm{pH}=2(2)$ & -12916800.62 & -7284369.69 & -10044823 & -10156697.28 & -349.98 \\
\hline $\mathrm{pH}=2(3)$ & -12916835.74 & -7284540.18 & -10044884.9 & -10156663.57 & -172.53 \\
\hline pH=2 (ave) & -12916823.59 & -7284467.38 & -10044886.06 & -10156738.23 & $-333.32 \pm 153.14$ \\
\hline $\mathrm{pH}=7(1)$ & -17333888.15 & -7284608.01 & -14463147.62 & -10155553.84 & -205.30 \\
\hline $\mathrm{pH}=7(2)$ & -17333883.25 & -7284531.8 & -14462891.9 & -10155339 & 184.20 \\
\hline $\mathrm{pH}=7(3)$ & -17334121.98 & -7284431.32 & -14462994.4 & -10155328.95 & 229.95 \\
\hline pH=7 (ave) & -17333964.46 & -7284523.71 & -14463011.29 & -10155407.26 & $69.62 \pm 239.18$ \\
\hline Ethanol (1) & -16173836.89 & -8000056.02 & -13303372.49 & -10870588.77 & -68.35 \\
\hline Ethanol (2) & -11617805.73 & -8000017.75 & -8746975.24 & -10870819.96 & 28.28 \\
\hline Ethanol (3) & -11617803.55 & -8000040.62 & -8746978.64 & -10870822.42 & 43.10 \\
\hline Ethanol (ave) & -13136482.06 & -8000038.13 & -10265775.46 & -10870743.72 & $1.01 \pm 60.52$ \\
\hline
\end{tabular}

\section{DISCUSSION}

Results of enthalpy calculations for vinpocetine release demonstrated that this process is an exothermal one as for release into water as into ethanol. The release into water in acidic medium ( $\mathrm{pH} 2$ ) proved to be the most intensive process since enthalpy of release into acidic medium is considerably less as compared with that one related with the release into neutral medium (Table 1). Probably, this is due to the fact that the cation of vinpocetine that is present at $\mathrm{pH} 2$ is more polar in a comparison with the base and so it has a higher affinity to water. 
Table 2. The results of quantum-chemical calculation of the release entropy for vinpocetine from sodium alginate into the solvent.

\begin{tabular}{|c|c|c|c|c|c|}
\hline \multirow[b]{2}{*}{ System } & \multicolumn{3}{|c|}{ Entropy of the system, $\mathbf{k J} / \mathbf{m o l}$} & & \multirow{2}{*}{$\begin{array}{l}\text { Released entropy } \\
\text { kJ/mol }\end{array}$} \\
\hline & $\begin{array}{l}\text { Vinpocetine in } \\
\text { polymer }\end{array}$ & Solvent & $\begin{array}{l}\text { Polymer without } \\
\text { vinpocetine }\end{array}$ & $\begin{array}{l}\text { Vinpocetine in the } \\
\text { solvent }\end{array}$ & \\
\hline $\mathrm{pH}=2$ & 1.7361 & 1.4434 & 1.6231 & 1.6059 & 0.0494 \\
\hline $\mathrm{pH}=2(2)$ & 2.1079 & 1.4232 & 1.6137 & 2.0306 & 0.1132 \\
\hline $\mathrm{pH}=2(3)$ & 2.1307 & 1.4068 & 1.7246 & 2.0036 & 0.1907 \\
\hline $\mathrm{pH}=2$ (ave) & 1.9916 & 1.4245 & 1.6538 & 1.8800 & $0.1178 \pm 0.0708$ \\
\hline $\mathrm{pH}=7(1)$ & 2.0070 & 1.4759 & 1.9391 & 1.6409 & 0.0971 \\
\hline $\mathrm{pH}=7(2)$ & 1.8528 & 1.4948 & 1.8779 & 1.9730 & 0.5033 \\
\hline $\mathrm{pH}=7$ (3) & 1.8011 & 1.5091 & 1.9557 & 1.9296 & 0.5752 \\
\hline $\mathrm{pH}=7$ (ave) & 1.8870 & 1.4933 & 1.9242 & 1.8478 & $0.3919 \pm 0.2578$ \\
\hline Ethanol (1) & 1.9313 & 1.9652 & 1.5261 & 1.7424 & -0.6281 \\
\hline Ethanol (2) & 2.0797 & 2.0143 & 1.7352 & 2.3872 & 0.0284 \\
\hline Ethanol (3) & 2.1415 & 1.9577 & 1.7438 & 2.3794 & 0.0240 \\
\hline Ethanol (ave) & 2.0508 & 1.9791 & 1.6684 & 2.1697 & $-0.1919 \pm 0.3778$ \\
\hline
\end{tabular}

Table 3. The results of quantum-chemical calculation of Gibbs energy for the release of vinpocetine from sodium alginate in the solvent.

\begin{tabular}{|c|c|c|c|c|c|}
\hline \multirow[b]{2}{*}{ System } & \multicolumn{2}{|c|}{ Gibbs energy, $\mathbf{k J} / \mathbf{m o l}$} & \multirow[b]{2}{*}{$\begin{array}{l}\text { Polymer without } \\
\text { vinpocetine }\end{array}$} & \multirow[b]{2}{*}{$\begin{array}{l}\text { Vinpocetine in the } \\
\text { solvent }\end{array}$} & \multirow{2}{*}{$\begin{array}{l}\text { Released energy of } \\
\text { Gibbs } \\
\text {, kJ/mol }\end{array}$} \\
\hline & $\begin{array}{l}\text { Vinpocetine in } \\
\text { polymer }\end{array}$ & Solvent & & & \\
\hline $\mathrm{pH}=2$ & -12917372.63 & -7284939.72 & -10045453.44 & -10157351.66 & -492.75 \\
\hline $\mathrm{pH}=2(2)$ & -12917454.06 & -7284810.89 & -10045323.26 & -10157326.77 & -385.08 \\
\hline $\mathrm{pH}=2(3)$ & -12917496.25 & -7284976.30 & -10045419.53 & -10157284.68 & -231.65 \\
\hline pH=2 (ave) & -12917440.98 & -7284908.97 & -10045398.74 & -10157321.04 & $-369.83 \pm 131.22$ \\
\hline $\mathrm{pH}=7(1)$ & -17334510.31 & -7285065.55 & -14463748.74 & -10156062.51 & -235.40 \\
\hline $\mathrm{pH}=7$ (2) & -17334457.61 & -7284995.19 & -14463474.00 & -10155950.64 & 28.16 \\
\hline $\mathrm{pH}=7$ (3) & -17334680.31 & -7284899.13 & -14463600.67 & -10155927.14 & 51.63 \\
\hline pH=7 (ave) & -17334549.41 & -7284986.62 & -14463607.80 & -10155980.10 & $-51.87 \pm 159.37$ \\
\hline Ethanol (1) & -16174435.61 & -8000665.24 & -13303845.58 & -10871128.92 & 126.35 \\
\hline Ethanol (2) & -11618450.44 & -8000642.18 & -8747513.15 & -10871560.01 & 19.47 \\
\hline Ethanol (3) & -11618467.42 & -8000647.50 & -8747519.22 & -10871560.05 & 35.65 \\
\hline Ethanol (cp.) & -13137117.82 & -8000651.64 & -10266292.65 & -10871416.33 & $60.49 \pm 57.61$ \\
\hline
\end{tabular}

From the results given in Table 2, it can be concluded that thermodynamic probability of vinpocetine occurrence in water is higher as compared with the polymer-bound state, implying that the entropy of release into this solvent is positive. Moreover, when the release from sodium alginate into ethanol takes place, on the contrary, thermodynamic probability is higher for vinpocetine-bound sodium, resulting in the negative release entropy.

According to the results for the calculated enthalpy of vinpocetine release, it can be concluded that this process is exothermic both for the release into water and into ethanol (Table 3). At the same time, release into water as an acidic medium ( $\mathrm{pH} 2$ ) is the most intensive process from the viewpoint of thermochemistry. Presumably, this is due to the fact that the cation of vinpocetine existing at $\mathrm{pH} 2$ is more polar than the base and has a higher affinity to water.

From the results of quantum chemical calculations on the enthalpy and entropy of the release of vinpocetine into solvents, it can be concluded that the release of vinpocetine from sodium alginate into water with $\mathrm{pH} 2$ is an energetically effective process. Moreover, it is accompanied by an increase in entropy, which indicates a higher thermodynamic probability of the final state (vinpocetine in the solution). The release of 
vinpocetine from sodium alginate into water at $\mathrm{pH} 7$ is energetically less efficient process as compared to the release into water with $\mathrm{pH} 2$ since enthalpy of release into the neutral medium is considerably less as compared with the enthalpy of release into acidic medium

The release of vinpocetine into ethanol from sodium alginate showed positive Gibbs energy, indicating a higher affinity of vinpocetine to sodium alginate in ethanol medium as compared to the aqueous medium. Presumably, this is due to the lower degree of swelling and dissociation of sodium alginate in ethanol compared to the aqueous medium. Because of this, the polymer is less polar, that can be the reason for a stronger binding of vinpocetine in the form of a base. In the water, the affinity of vinpocetine to sodium alginate is higher in the neutral medium ( $\mathrm{pH} 7)$ and lower for the acidic medium ( $\mathrm{pH} 2)$.

According to the obtained data on thermodynamic characteristics of vinpocetine release one can make a suggestion that the highest release of this substance from sodium alginate into water has occurred in the acidic environment at $\mathrm{pH} 2$.

\section{CONCLUSION}

Quantum chemistry method was used to study the release of vinpocetine from natural sodium polysaccharide alginate into the following media: water with $\mathrm{pH} 2$ and 7;95\%-ethyl alcohol. It was found that the release of vinpocetine is most energetically prreferable into water with $\mathrm{pH} 2$ as compared to the value of $\mathrm{pH} 7$ and 95\%-ethyl alcohol. The obtained results coincide with the results of the molecular dynamics modeling of vinpocetine release from the sodium alginate and allow to recommend this polymer for application as a film-former material.

\section{MATERIALS AND METHODS}

The initial state for calculating thermodynamic characteristics of vinpocetine release from the polymer was taken as the conformation of the "polymer-vinpocetine" system after its thermodynamic balancing by the molecular dynamics method in the BioEvrik program for the period of $5 \mathrm{~ns}[18,19]$. As the final state, the instant of molecular dynamics simulation was applied, corresponding to the completely released vinpocetine molecule from the polymer into solvent. As a criterion for vinpocetine released into solvent, the energy modulus of the van der Waals interaction between the vinpocetine molecule and the polymer $(|\mathrm{E}|<0.1$ $\mathrm{kJ} / \mathrm{mol}$ ) was applied [20]. For both states, geometry optimization was carried out in the Amber 94 force field in the BioEvric program.

For the isolated systems, an oscillatory analysis was performed using the unrestricted Hartree-Fock method in the STO-3G basis applying Orca 4.0 program [21]. As a result, thermodynamic characteristics of the modeled fragments for the system (enthalpy and entropy) were obtained. Further, the enthalpy $(\Delta \mathrm{H})$ and entropy $(\Delta S)$ of the vinpocetine release from the polymer were calculated using the following formulae:

(Eq. 1) $\Delta H=\left(H_{\text {polymer }}+H_{\text {vinpocetine-solvent }}\right)-\left(H_{\text {vinpocetine-polymer }}+H_{\text {solvent }}\right)$

(Eq.2) $\Delta S=\left(S_{\text {polymer }}+S_{\text {vinpocetine-solvent }}\right)-\left(S_{\text {vinpocetine-polymer }}+S_{\text {solvent }}\right)$

Gibbs energies $(\Delta G)$ of vinpocetine release into various media were calculated using the obtained enthalpies and entropies; the calculation was carried out using the following formula at the temperature of $310 \mathrm{~K}$ :

(Eq.3) $\quad \Delta \mathrm{G}=\Delta \mathrm{H}-(\mathrm{T} \Delta \mathrm{S})$

Where, $\mathrm{T}$ - temperature $(\mathrm{K})$.

Acknowledgements: The authors thank of the grant of the Council on the grants of the President of RF CП-95.2018.4.

Author contributions: Concept -Y.P., K.K.; Design - Y.P., K.K., H.A.J; Supervision - Y.P., K.K.; Resource Y.P., K.K.; Materials - Y.P., K.K.; Data Collection and/or Processing - Y.P., K.K., H.A.J; Analysis and/or Interpretation - Y.P., K.K.; Literature Search - Y.P., K.K., H.A.J; Writing - Y.P., K.K.; Critical Reviews - Y.P., K.K.

Conflict of interest statement: The authors declared no conflict of interest. 


\section{REFERENCES}

[1] Aubert-Pouessel A. Preparation of PLGA of PLGA microparticles by an emulsionextraction process using glycofurol as polymer solvent. Pharm Res. 2004; 21: 2384-2391.

[2] Jachowicz RE, Nürnberg E, Pieszczek B, Kluczykowska B, Maciejewska A. Solid dispersion of ketoprofen in pellets. Int J Pharmaceut. 2000; 206(1): 13-21.

[3] Senuma Y, Lowe C, Zweifel Y, Hilborn JG, Marison I. Alginate hydrogel. microspheres and microcapsules prepared by spinning disk atomization. Biotechnol Bioeng. 2000; 67: 616-622.

[4] Sugiura S, Oda T, Izumida Y, Aoyagi Y, Satake M, Ochiai A, Ohkohchi N, Nakajima M. Generation of monodisperse alginate microbeads and in situ encapsulation of cell in microfluidic device. Biomaterials. 2005; 26: 3327-3331.

[5] Silva MP, Tulini FL, Marcela MR, Penning M, Fávaro-Trindade CS, Poncelet D. Microcapsules loaded with the probiotic Lactobacillus paracasei BGP-1 produced by co-extrusion technology using alginate/shellac as wall material: Characterization and evaluation of drying processes. Food Res Int. 2016; 89(1): 582-590.

[6] Ding J, Li J, Shirui Mao. Development and evaluation of vinpocetine inclusion complex for brain targeting. Asian J Pharm Sc. 2015; 10: 114-120.

[7] Polkovnikova YA, Slivkin AI. Vinpocetine release from a microencapsulated form. Pharm Chem J. (Khimikofarmatsevticheskii zhurnal) 2016; 50(8): 553-555.

[8] Polkovnikova YA. Studies on the development of encapsulated formulations of vinpocetıne. Russ J Biopharmaceut. 2015; 7(4): 31-36.

[9] Hills AG. PH and the HENDERSON-HASSELBALCH equation. Am J Med. 1973; 55: 131 - 133.

[10] Miyamoto H, Rein DM, Kazuyoshi U, Yamane C, Cohen Y. Molecular dynamics simulation of cellulose-coated oilin-water emulsions. Cellulose. 2017; 24(7): 2699 - 2711.

[11] Hui-dong Zheng, Wu F, Wang B, Wu Y. Molecular dynamics simulation on the interfacial features of phenol extraction by tbp/dodecane in water. Comput Theor Chem. 2011; 970: 66-72.

[12] Clare BW, Supuran CT. Semi-empirical atomic charges and dipole moments in hypervalent sulfonamide molecules: descriptors in qsar studies. J Mol Struct (Theochem). 1998; 428: 109 - 121.

[13] Brian J. Teppen Hyperchem, release 2: molecular modeling for the personal computer. J Chem Inf Comput Sci. 1992; 32: 757 - 759 .

[14] Devlin PJ, Finley JW, Stephens PJ, Frisch MJ. Ab initio calculation of vibrational absorption and circular dichroism spectra using density functional force fields: a comparison of local, nonlocal, and hydrid density functionals. J Phys Chem. 1994; 98: 11623-11627.

[15] Cornell WD, Cieplak P, Bayly CI, Gould IR, Merz KM, Ferguson DM, et al. A second generation force field for the simulation of proteins, nucleic acids, and organic molecules. J Am Chem Soc. 1995; 117: 5179-5197.

[16] Berendsen HJC, Postma JPM, Gunsteren WF, DiNola A, Haak JR. Molecular dynamics with coupling to an external bath. J Chem Phys. 1984; 81: 3684-3690.

[17] Bykov D, Petrenko T, Izsák R, Kossman S, Becker U, Neese F. Efficient implementation of the analytic second derivatives of hartree-fock and hybrid dft energies: a detailed analysis of different approximations. Mol Phys. 2015; 113: 1961.

[18] Greiner M, Sonnleitner B, Mailänder M, Briesen H. Modeling complex and multi-component food systems in molecular dynamics simulations on the example of chocolate conching. Food Funct. 2014; 5: 235-242.

[19] Feller SE. Molecular dynamics simulations of lipid bilayers. Curr Opin Colloid Interface Sci. 2000; 5: 217-223.

[20] Leo D, Maranon J. Confined water/oil interface. Molecular dynamics study. J Mol Struct (Theochem). 2004; 672: 221229.

[21] Sedghi M, Piri M, Goual L. Atomistic molecular dynamics simulations of crude oil/brine displacement in calcite mesopores. Langmuir. 2016; 32: 3375-3384. 